\title{
Glottodidaktische Steuerung und Regelung auf interindividueller Ebene
}

\author{
Glottodidactic control and regulation \\ on the inter-individual level
}

\begin{abstract}
AвSTRACT. The subject of this discussion consists of selected concepts regarding the codematic foundations of foreign-language learning and cybernetic systems of foreign-language communication. The author focuses on processes of controlling, regulating, and directing foreign-language structures at the inter-individual level from the teacher to the learner. The control process, coupled with the process of basic information transfer, supports and strengthens it, both with linguistic (suprasegmental) means and extra-linguistic (visual) means. The basis of the regulation process are the effects of the coded structures of the learner, effects delivered to the teacher by the control system functioning through feedback. Glottodidactic direction includes the above two processes in order to transfer inaccurate structures, once corrected by the teacher, with the aim of re-decoding them and correctly assimilating them.

KEYWORDS: control of information signals, regulation of information structures, glottodidactic direction, communication partner, the participant in foreign-language learning.

SCHLÜSSELWÖRTER: Steuerung der Informationssignale, Regelung der Informationsstrukturen, glottodidaktische Lenkung, fremdsprachlicher Kommunikationspartner und fremdsprachlicher Unterrichtsteilnehmer.
\end{abstract}

\section{PROBLEMSTELLUNG}

Die im Titel angeführten Prozesse sind für die Glottodidaktik relevant, weil sie in bestimmten Zusammenhängen direkt und indirekt mit der Darbietung und der Erlernung des fremdsprachlichen Materials stehen. Gerade auf diesen Standpunkt soll im Weiteren näher eingegangen werden. Unsere Erörterungen basieren auf den von Zabrocki aufgestellten und ausgebauten Theorien über die kodematischen Grundlagen des Fremdsprachenunterrichts und die kyber- 
netischen Konzepte der (fremd-)sprachlichen Kommunikation (Zabrocki 1966 u. 1975).

Aufgrund der bereits angedeuteten Theorien wird ein kodematisch-glottodidaktisches Modell konzipiert, das den Sprechvorgang zwischen dem Fremdsprachenlehrer und dem Fremdsprachenlerner wiedergibt. Es kann folgendermaßen dargestellt werden (Abb. 1.).

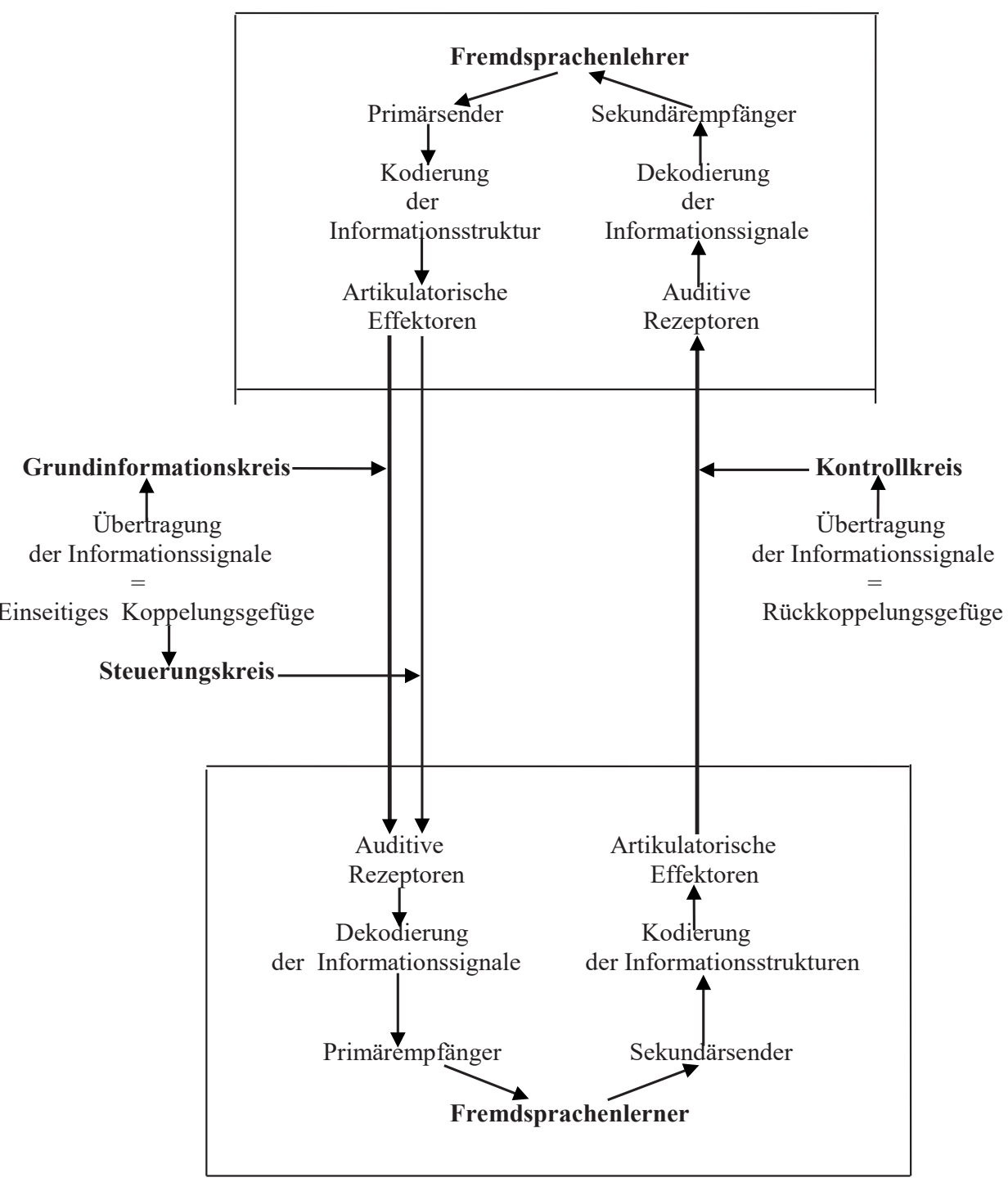

Abbildung 1. Glottodidaktische Kommunikationsprozesse zwischen dem Fremdsprachenlehrer und dem / den Fremdsprachenlerner(n) 


\section{DIE STEUERUNG UND IHRE WESENTLICHEN MITTEL}

Als Gegenstand der kybernetischen Begriffsbestimmungen gelten unter anderem Steuerung, Regelung und Rückkopplung, die in den Übertragungsprozessen der sprachlichen Informationen verwirklicht werden. Unseren Überlegungen zugrunde liegen die glottodidaktischen Kommunikationsvorgänge, die sich zwischen dem Lehrenden und den Lernenden vollziehen. Es sind also ablaufende Kodierungsprozesse der Informationsstrukturen auf der intraindividuellen Ebene des Lehrenden, Übertragungsprozesse der Informationssignale auf der interindividuellen Ebene zwischen den Kommunikationspartnern und deren Dekodierungsprozesse auf der intraindividuellen Ebene des / der Lernenden.

Mit den Übertragungsprozessen der Informationssignale auf der interindividuellen Ebene laufen räumlich und zeitlich parallel die Steuerungsprozesse ab (s. Abb. 1.), da jede Informationsübertragung eine Steuerung voraussetzt, d.h., dass die sich im Grundinformationskreis und im Steuerungskreis vollziehenden Vorgänge eng miteinander verbunden sind und sich gegenseitig bedingen (vgl. Zabrocki 1966: 8; Schaefer 1972: 95 f.).

Wesentlich für die Glottodidaktik sind die zu lehrenden und zu lernenden fremdsprachlichen Strukturen, welche die außersprachliche Wirklichkeit auf spezifische Art und Weise abbilden, und dementsprechend dargeboten und erlernt / angeeignet werden müssen. Die Spezifik bezieht sich auf diejenigen Fremdsprachenstrukturen, die sich von denen der Muttersprache der Lernenden sowohl im Ausdrucksplan als auch im Inhaltsplan unterscheiden. Und gerade deshalb ist es wichtig, die zu lernenden Fremdsprachenstrukturen so darzulegen und zu erklären, dass sie durch die Lernenden richtig empfangen und dekodiert, d.h. kennengelernt und verstanden werden sowie im fremdsprachlichen Speicher-Mechanismus als Strukturmatrizen konstruiert und für gewisse Zeit behalten / gespeichert werden. Um solche Ergebnisse erreichen zu können, bedient man sich bei der Informationsübertragung der Steuerung, welche in den glottodidaktischen Kommunikationssituationen über zwei Arten von Grundmitteln verfügt. Zum einen sind es sprachliche Mittel (vgl. die suprasegmentale Funktion der Betonung, der Intonation und des Rhythmus in den Sprechakten), zum anderen außersprachliche bzw. visuelle Mittel, welche durch die optische Darbietung von Gegenständen, Erscheinungen oder Situationen deren semantische oder grammatische Verständlichkeit erleichtern (vgl. ausführlicher Szczodrowski 2001: 47-82).

Die Übertragung der Informationssignale beeinflusst sowohl äußere als auch innere Störungen, die auf diesen Prozess bestimmte negative Konsequenzen haben und vor allem Aufnahme- sowie Verarbeitungs- und Speicherungsschwierigkeiten verursachen können. Darüber hinaus geht es um die Hervorhebung 
der potentiellen Steuerungskraft und deren Wirkung, welche die während der Übertragung der akustischen oder der optischen Informationssignale häufig vorkommenden Störungen abschwächen oder ganz beseitigen können. Es dürfte jedoch selbstverständlich sein, dass von den Bedingungen der Übertragung der Informationssignale direkt die Aufnahme- und Dekodierungsfähigkeit der auditiven und visuellen Rezeptoren des Empfängers abhängig sind. In den Rezeptoren werden die phonetisch-phonologischen oder die graphisch-graphematischen Strukturen dekodiert, aber deren gänzliche, d.h. semantisch-grammatische Dekodierung findet im fremdsprachlichen Speicher-Mechanismus statt.

Zusammenfassend bleibt festzuhalten, dass dieSteuerung gekoppelt mit den kodierten Informationssignalen, die auf der individuellen Ebene vom Primärsender zum Primärempfänger übermittelt werden, als einseitiger, offener und zielgerichteter Vorgang aufzufassen ist.

Von wesentlicher Bedeutung für den fremdsprachlichen Unterricht sind zwei Probleme: Erstens die schon erlernten Strukturen, die als Strukturmatrizen den Speicher-Mechanismus bilden, weiter zu festigen und möglicherweise ins Langzeitgedächtnis einzukodieren. Zweitens, die zum ersten Mal empfangenen Strukturen zu dekodieren, damit sie richtig kennengelernt und gleichzeitig als phonetisch-phonematische oder graphisch-graphematische und semantisch-grammatische Strukturmatrizen konstruiert und für eine gewisse Zeit im Speicher-Mechanismus behalten werden. Aus der bereits formulierten Konstatierung lässt sich erkennen, dass in solch einem Unterrichtsprozess zwei weitere Probleme auftauchen. Sie betreffen und stecken in den Dekodierungsfähigkeiten des Empfängers / Lernenden bezüglich der bekannten und der neu zu lernenden Strukturen. Es kommen eigentlich zwei verschiedene Dekodierungsprozesse vor, und zwar bezieht sich der eine auf die bekannten Strukturen, der andere auf die neuen. Diese Prozesse sind qualitativ und quantitativ , unterschiedlich', und deshalb kann man deren Verlauf keinesfalls ex aequo auffassen. Der allgemeine Grund hierfür besteht in der Tatsache, dass die bekannten Strukturen als Matrizen im Speicher-Mechanismus schon existieren und deren Dekodierung auf der Zuordnung der Strukturen zu den entsprechenden Sprachmatrizen beruht. Im Falle der neu empfangenen Strukturen werden sie einerseits dekodiert und andererseits in Form von Strukturmatrizen konstruiert, wodurch sie das lexikalisch-grammatische Inventar des Speicher-Mechanismus des Lernenden bereichern.

Die oben getroffenen Unterscheidungen zwischen den von den Lernenden gespeicherten Strukturen und denen, die gerade von ihnen dekodiert, kennengelernt und gespeichert werden, beziehen sich direkt auf die glottodidaktischen Handlungen, welche wie folgt differenziert und festgelegt werden: 
1. Die bekannten Informationsstrukturen werden von den Lernenden dekodiert und denen im Speicher-Mechanismus existierenden Strukturmatrizen zugeordnet. Es kann mit hoher Wahrscheinlichkeit angenommen werden, dass die Lernenden während des Dekodierungsvorgangs der Strukturen als Kommunikationspartner fungieren.

2. Die neu empfangenen Informationsstrukturen werden von den Lernenden dekodiert, kennengelernt und gleichzeitig in Form von Strukturmatrizen im Ultrakurzzeitgedächtnis oder im Kurzzeitgedächtnis konstruiert und dort für eine bestimmte Zeit gespeichert. In solch einem Falle hat man es mit Prozessen zu tun, die sensu strictiore einen glottodidaktischen Charakter haben, und dementsprechend sind die Lernenden als tätige Unterrichtsteilnehmer zu betrachten.

Aus den bereits erfassten Unterscheidungen erfolgt die Feststellung, dass die im glottodidaktichen Prozess den Empfängern übertragenen Strukturen in zwei Gruppen eingeteilt werden können: Einerseits sind es die erlernten und die gespeicherten Strukturen, welche über ihre rezeptiv-produktiven Fähigkeiten verfügen, andererseits die zu lernenden, welche genauerer Dekodierung und grundlegender Speicherung bedürfen. Auf diese Weise steht dem Lernenden ein doppelter Dekodierungsvorgang bevor; der eine bezieht sich auf die kommunikativen Aspekte der Strukturen, der andere dagegen betrifft die glottodidaktischen Strukturen.

\section{DIE REGELUNG UND IHRE AUFGABEN}

Der Regelungsprozess, welcher auf dem rückgekoppelten Kontrollkreis basiert, findet auf der interindividuellen Ebene vom Sekundärsender zum Sekundärempfänger statt, d.h. der Regelung liegen die durch den Sekundärsender kodierten Informationsstrukturen zugrunde. In diesem Falle handelt es sich um die direkten fremdsprachlichen Sprechreaktionen des Lernenden auf die durch den Lehrenden zugesandten Informationen, unter denen die neu kennengelernten lexikalischen oder grammatischen Strukturen in bekannte syntagmatischsyntaktische Strukturkonstruktionen eingefügt worden sind. Der Lehrende will erfahren, wie der Lernende die neu empfangenen und kennengelernten Strukturen gelernt / behalten hat und ob er imstande ist, sie zu (re-)produzieren. Hier muss nochmals betont werden: Es geht zum einen um die Fähigkeiten des Fremdsprachenlernenden bezüglich der Aufnahme, der Dekodierung und der Speicherung der neu empfangenen Strukturen in Form von Sprachmatrizen, zum anderen um seine Kodierungspotentialität und deren Ergebnisse. Im ersten 
Fall gehört es zur Aufgabe des Lehrenden, die Stabilität der bereits konstruierten Strukturmatrizen im Speicher-Mechanismus des Fremdsprachenlernenden und deren Kodierungsfähigkeiten approximativ einzuschätzen, im zweiten Fall muss er insbesondere seine Kodierungsergebnisse bewerten. Werden bestimmte Strukturen als unkorrekt oder mangelhaft beurteilt, dann bedürfen sie zunächst einer genauen Regelung, des Weiteren einer erneuten interindividuellen Übertragung an den Lernenden und darüber hinaus einer zusätzlich durchdachten sprachlichen und / oder außersprachlichen Steuerung, damit sie korrekt behalten werden können.

Dank der Rückkopplung werden die Kodierungsergebnisse des Lernenden / Sekundärsenders vom Primärsender / Lehrenden als Rückmeldungen empfangen. Es handelt sich hierbei also um direkte zurückgemeldete Informationen des Lernenden, die mit den vorherübermittelten Informationen des Lehrenden zuerst verglichen, dann beurteilt und bei Bedarf korrigiert werden. Auf diese Art und Weise eröffnet sich eine neue Informationsübertragung mit den schon verbesserten Strukturen, die der Lernende richtig dekodieren muss, wobei er die mangelhafte Strukturmatrize vervollkommnet (s. auch Schröder 1975: 31 f.). In solch einem Falle haben wir es mit einem weiteren, dem geregelten Kommunikationsprozess zu tun, der ein Nachkonstruktionsprozess der Strukturmatrize(n) im Speicher-Mechanismus des Lernenden ist, welcher bewirkt, dass die Strukturmatrize(n) den in der zu lernenden Fremdsprache geltenden Gesetzmäßigkeiten entspricht (entsprechen).

\section{KOMPLEXITÄT DER GLOTTODIDAKTISCHEN LENKUNG}

In den kybernetischen Grundsatzfragen werden die Steuerung und die Regelung der Informationen unter dem Begriff Lenkung zusammengefasst, wobei die Steuerung als offene und einseitige Wirkungskette betrachtet wird, die Regelung dagegen als geschlossener Wirkungskreis, der durch die Rückkopplung, d.h. durch einen kreisförmigen Prozess entsteht und abläuft (vgl. Lerner 1970: 96 ff.; Maser 1971: 169; Klaus \& Liebscher 1974: 92 f.; Sachsse 1974: 99; Burkart 1983: 47). Was die Glottodidaktik anbetrifft, so werden die beiden auf der interindividuellen Ebene stattfindenden Übertragungsprozesse von Informationen - und zwar die Regelung vom Sekundärsender zum Sekundärempfänger und die Steuerung vom Primärsender zum Primärempfänger - in den Lenkungsprozess eingegliedert. Dementsprechend muss sich der Lehrende insbesondere auf folgende Aufgaben konzentrieren:

1. Die durch den Lernenden fehlerhaft und mangelhaft kodierten Fremdsprachenstrukturen zu korrigieren und sie erneut an ihn zu übertragen. 
2. Eine bessere sprachliche oder außersprachliche Steuerung der zu übertragenden Informationsstrukturen, damit die Empfänger sie einwandfrei rezipieren und richtig dekodieren.

3. Überlegungen zur Frage, ob die Lernenden noch zusätzliche lexikalische oder grammatische Kenntnisse über die korrekten Fremdsprachenstrukturen brauchen, die ihnen behilflich wären, diese gut zu verstehen und richtig zu gebrauchen.

Die Komplexität der Lenkungsprozesse, die einerseits die erfolgreiche Erlernung der Fremdsprachenstrukturen und andererseits das Korrigieren der fehlerhaft vorkommenden Strukturen begünstigt, kann man als übergeordnete prozessuale fremdsprachenunterrichtliche Ebene auffassen, die nicht nur die intraindividuellen Rezeptions-, Dekodierungs-, Speicherungs-, Verarbeitungs- und Kodierungsfähigkeiten des Lernenden, sondern auch die möglichen glottodidaktischen Lehr- und Lernmethoden berücksichtigt. Gemeint sind hier die klare Darbietung und die genaue Übertragung des zu lernenden Materials, d.h. solche Prozesse, die immer eine Steuerung verlangen, um die neu eingeführte lexikalische oder grammatische Struktur besonders hervorzuheben, was deren Rezeption und Dekodierung dieses Materials sicherlich erleichtert.

Die durch die Fremdsprachenlernenden kodierten Strukturen, unter denen sich fehlerfreie und auch fehlerhafte befinden, erscheinen bekanntlich im rückgekoppelten Kontrollgefüge. Die fehlerfreien Strukturen werden vom Lehrenden selbstverständlich akzeptiert, weil sie dem fremdsprachlichen Ist-Wert entsprechen, die fehlerhaften dagegen werden korrigiert, wiederholend dargestellt und übertragen, damit der Lernende sie richtig dekodiert und so speichert, dass sie mit deren Soll-Wert-Zustand übereinstimmen.

Auf diese Art und Weise ist die Regelung ebenso wie die Steuerung als zielgerichteter Prozess zu begreifen, wobei die zurückgemeldeten Kodierungserfolge des Lernenden ständig kontrolliert und oft auch verbessert werden (müssen). Die Komplexität der glottodidaktischen Lenkung kann man auch so verstehen, dass dieser Vorgang sowohl mit der Nachkonstruktion als auch mit der beispielsweise semantischen, syntagmatischen oder syntaktischen Verarbeitung (vgl. zum Beispiel Schnabl 1972: 69 u. 104 ff.) der schon im fremdsprachlichen Speicher-Mechanismus existierenden, aber noch nicht richtig aufgebauten und funktionierenden Strukturmatrizen, eng verbunden ist. Die genauere Betrachtung der Verarbeitung führt jedoch zur Spezifizierung, dass in diesem Prozess mögliche Verknüpfungen der gerade neu entschlüsselten Struktur mit anderen gespeicherten Strukturen zustande kommen können. Unsere bisher angestellten Erörterungen über die Fragen der zusammengesetzten Lenkungsprozesse lassen sich graphisch folgendermaßen schildern: 


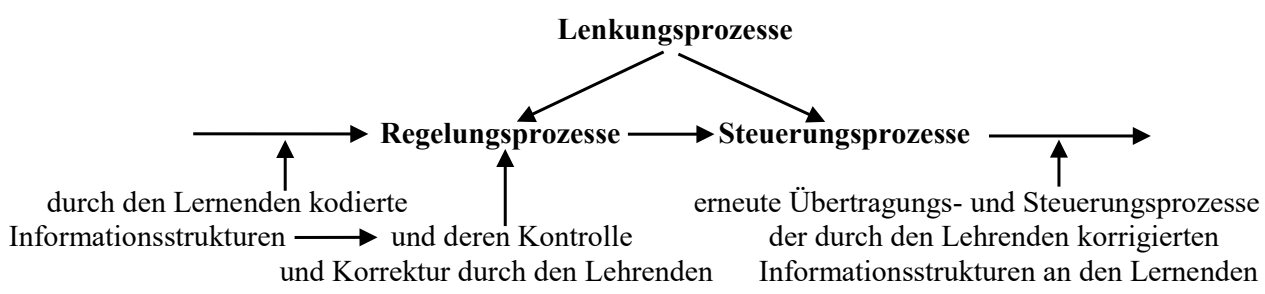

Abbildung 2. Lenkungsprozesse und ihre grundlegenden Bestandteile: Regelungsprozesse und Steuerungsprozesse

Da am Fremdsprachenunterricht immer eine bestimmte Anzahl von Lernenden teilnimmt, ergeben sich auch Probleme anderer Art, und zwar sollten immer die individuelle Sprachbegabung und Sprachleistung jedes Lernenden berücksichtigt werden. Dementsprechend müssen sowohl allgemeine glottodidaktische Ziele beachtet werden als auch solche, die nur einige Unterrichtsteilnehmer betreffen, die beispielsweise mehr Zeit benötigen, das fremdsprachliche Material zu erlernen. Es stellt sich dennoch die Frage: Wie viele Male muss der einzelne Schüler bestimmte Strukturen üben, um sie sich einzuprägen und richtig zu bewältigen. Solche Aufgaben stehen im Mittelpunkt glottodidaktischer Lenkungsprozesse.

\section{SCHLUSSFOLGERUNGEN}

Durch unsere abschließenden Bemerkungen soll noch einmal betont werden, dass sich der fremdsprachliche Unterricht vom theoretischen und praktischen Standpunkt aus unter zwei Aspekten betrachten lässt: Erstens sind die dargebotenen Strukturen zum größten Teil schon bekannt, und der Lernende dekodiert sie auf solche Weise, dass sie durch die rezeptive Dynamisierung der gespeicherten Strukturmatrizen abgerufen, ganzheitlich dekodiert und dabei auch gefestigt, d.h. immer tiefer ins Gedächtnis (in den Speicher-Mechanismus) eingeprägt / einkodiert werden. Zweitens befinden sich unter den übermittelten Strukturen auch solche, die zum ersten Mal präsentiert, kennengelernt und erlernt werden. Diesen Strukturen wird während deren Übertragung, Steuerung und Aufnahme besondere Aufmerksamkeit geschenkt, damit sie richtig dekodiert und als fremdsprachliche Strukturmatrizen konstruiert und gespeichert werden können. 


\title{
LITERATURVERZEICHNIS
}

Burkart, R. (1983). Kommunikationswissenschaft. Wien / Köln: Böhlau Verlag.

Klaus, G. / Liebscher, H. (1974). Systeme, Informationen, Strategien. Eine Einführung in die kybernetischen Grundgedanken der System- und Regelungstheorie, Informationstheorie und Spieltheorie. Berlin: VEB Verlag Technik.

Lerner, A. Ja. (1970). Grundzüge der Kybernetik. Braunschweig: Vieweg Verlag.

Maser, S. (1971). Grundlagen der allgemeinen Kommunikationstheorie. Stuttgart u.a.: Verlag W. Kohlhammer.

Sachsse, H. (1974). Einführung in die Kybernetik unter besonderer Berücksichtigung von technischen und biologischen Wirkungsgefügen. Braunschweig: Verlag Vieweg.

Schaefer, G. (1972). Kybernetik und Biologie. Stuttgart: J.B. Metzlersche Verlagsbuchhandlung.

Schnabl, H. (1972). Sprache und Gehirn - Elemente der Kommunikation. Zu einem kybernetischen Modell der menschlichen Nachrichtenverarbeitung. München: Wilhelm Goldmann Verlag.

Schröder, H. (1975). Kommunikation und Information im Unterricht. KG München: Franz Ehrenwirth Verlag.

Szczodrowski, M. (2001). Steuerung fremdsprachlicher Kommunikation. Gdańsk: Wydawnictwo Uniwersytetu Gdańskiego.

Szczodrowski, M. (2007). Zur Struktur und Funktion des fremdsprachlichen Rückkopplungsgefüges. In: K. Grzywka / J. Godlewicz-Adamiec / M. Grabowska / M. Kosacka / R. Małecki (Hrsg.), Kultura - Literatura - Język. Festschrift für Herrn Professor Lech Kolago zum 65. Geburtstag (S. 828-836). Warszawa: Instytut Germanistyki Uniwersytetu Warszawskiego.

Zabrocki, L. (1966). Kodematische Grundlagen der Theorie des Fremdsprachenunterrichts. Glottodidactica. An International Journal of Applied Linguistics, I, 3-42.

Zabrocki, L. (1975). Kybernetische Modelle der sprachlichen Kommunikation. Wrocław / Warszawa / Kraków / Gdańsk: Zakład Narodowy im. Ossolińskich / Wydawnictwo Polskiej Akademii Nauk / Komitet Neofilologiczny.

Received: 02.01.2019; revised: 30.01 .2020

\author{
MARIAN SZCZODROWSKI \\ Ateneum Szkoła Wyższa w Gdańsku \\ m.szczodrowski6@upcpoczta.pl \\ ORCID: 0000-0003-4881-8226
}


